\section{OC-039 EPS8: A MASTER REGULATOR OF INTEGRIN-DEPENDENT PANCREATIC CANCER INVASION}

doi:10.1136/gutjnl-2013-304907.039

1,"J Tod, 'V Jenei, 'M Chrzan, 'C Johnson, 'D Fine, 'G J Thomas. 'Cancer Sciences, University of Southampton School of Medicine, Southampton, UK

Introduction EGFR pathway substrate 8 (Eps8) is an adapter protein that sits at the heart of a complex system regulating re-organisation of the actin cytoskeleton. Eps8 has been shown to be involved in cell motility and EGFR internalisation, via interactions with multiple intracellular proteins, including integrins. $\alpha v \beta 6$ is an epithelial specific integrin, not present in healthy tissue but overexpressed in many carcinomas. $\alpha v \beta 6$ promotes tumour invasion and is a key activator of TGF $\beta$, which modulates tumour cell EMT and the desmoplastic response characteristically present in PC. The aim of this study was to investigate whether there is a link between Eps8 and $\alpha v \beta 6$ in pancreatic cancer invasion.

Methods We used immunochemistry to examine the expression of Eps 8 and $\alpha v \beta 6$ in normal pancreas and PC in vivo using tissue microarrays. PC cell motility was investigated using Transwell@ assays. siRNA interference was used to down-regulate genes of interest. The ability of PC cells to activate TGF $\beta$ was measured using TGF $\beta$-responsive mink lung epithelial cells. Cell surface $\alpha v \beta 6$ levels were assessed with FACS analysis. Endocytosis of $\alpha v \beta 6$ was suppressed using Clathrin siRNA to examine the role of endocytosis in $\alpha v \beta 6$ functions. Pancreatic stellate cells (PSCs) were isolated from primary pancreatic resection tissue and used to optimise 3D pancreatic organotypic cultures.

Results Eps 8 and $\alpha v \beta 6$ were up-regulated in $>70 \%$ PC in vivo but were not generally detected in normal pancreas. An organotypic model of pancreatic cancer was developed using primary PSCs to study $\alpha v \beta 6$ functions. $\alpha v \beta 6$ promoted PC cell invasion and TGF $\beta$ activation. Interestingly, Eps8 knockdown suppressed $\alpha v \beta 6$-dependent motility but conversely, promoted $\alpha v \beta 6$ dependent TGF $\beta$ activation. Although inhibition of $\alpha v \beta 6$ endocytosis also increased TGF $\beta$ activation, this was not Eps8 dependent, nor did Eps8 knockdown affect total cell surface levels of the integrin.

Conclusion $\alpha v \beta 6$ and Eps 8 are over-expressed in PC. $\alpha v \beta 6$ promotes both invasion and TGF $\beta$ activation, and Eps8 appears to act as a molecular switch between these functions. How this relates to disease progression is unclear; in late stage disease, where canonical TGF $\beta$ signalling is dysregulated (e.g. SMAD4 mutations which are seen in $>50 \%$ of PCs) both $\alpha v \beta 6$ functions are likely to be tumour promoting. However, in premalignancy when canonical TGF $\beta$ signalling acts as a tumour suppressor, we speculate that up-regulation of Eps8 may result in a pro-invasive phenotype and facilitate tumour development. Eps8 may therefore act as a master regulator of PC invasion by switching the cell function between motility and TGF $\beta$ activation at critical time points in PC progression.

Disclosure of Interest None Declared

\section{OC-040 WITHDRAWN BY AUTHOR}

\section{OC-041 ENHANCED MONOCYTE FUNCTION AND SURFACE TLR EXPRESSION PREDICT POST-OPERATIVE SYSTEMIC INFLAMMATORY RESPONSE SYNDROME IN PATIENTS IMMEDIATELY FOLLOWING MAJOR HEPATIC AND PANCREATIC RESECTION}

doi:10.1136/gutjnl-2013-304907.040

1."R Lahiri, 'J Wong, ${ }^{2} \mathrm{~S}$ Bhattacharya, ' 'G Foster, 'W Alazawi. 'Centre for Digestive Diseases, Queen Mary, University of London; ${ }^{2}$ Barts Health HPB Centre, Royal London Hospital, London, UK
Introduction Patients undergoing major surgery are at risk of systemic inflammatory response syndrome (SIRS) and life-threatening post-operative sepsis. Early identification of high-risk patients would allow for tailored post-operative care. We tested the hypothesis that post-operative SIRS could be predicted by differences in innate immune function in peripheral blood mononuclear cells (PBMC) taken from patients undergoing major abdominal surgery in the perioperative period.

Methods Serial blood samples were taken from consenting, adult patients undergoing major hepatic or pancreatic surgery preoperatively and on days one and two postoperatively. Patients with inflammatory co-morbidities, pre-operative sepsis and those taking anti-inflammatory or immunomodulatory medication were excluded. PBMCs were isolated, stimulated for 24 hours with lipopolysaccharide (LPS) or flagellin and cytokine production was quantified by ELISA. PBMC surface expression of CD14, TLR4 and TLR 5 was assessed by flow cytometry. SIRS was defined by the American College of Chest Physicians/Society of Critical Care Medicine consensus criteria.

Results We included 32 patients, nine of whom developed SIRS on day five or later. There were no significant pre-operative differences between the SIRS and the non-SIRS groups in TLR-induced IL- 6 and TNFa production nor TLR4 and TLR5 expression. LPSand flagellin- induced IL-6 concentrations were significantly greater in the SIRS group on day two $(1532 \mathrm{pg} / \mu \mathrm{l}$ vs $153 \mathrm{pg} / \mu \mathrm{l} ; \mathrm{p}<$ 0.0002). Monocyte expression of both TLR4 and TLR5 was significantly greater in the SIRS group on both day one $(p<0.002)$ and day two $(p<0.001)$. There was no significant difference in CRP or other clinical predictors of SIRS between the groups in the early post-operative period.

Conclusion Patients who develop SIRS show increased cytokine production in response to TLR stimulation in the early post-operative period. This is associated with elevated surface TLR4 and TLR5 expression in monocytes. These data indicate that there are early functional differences in innate immune responses in patients who develop SIRS compared to those who do not, and that these differences are detectable days before the onset of clinical symptoms. Such assays can help to identify at-risk patients in whom intensive therapy can be targeted Disclosure of Interest None Declared

\section{OC-042 ENDOSCOPIC ULTRASOUND GUIDED FINE NEEDLE ASPIRATION OF PANCREATIC MASS LESIONS CAN BE LESS ACCURATE WITH A METALLIC STENT IN SITU BUT SHOULD NOT DELAY TREATMENT OF OBSTRUCTIVE JAUNDICE}

doi:10.1136/gutjnl-2013-304907.041

1,*A D Hopper, ${ }^{2} \mathrm{R}$ Vinayagam, ${ }^{3} \mathrm{~A}$ Dube. 'Department Gastroenterology, Royal Hallamshire Hospital; '2Department of Radiology, Northern General Hospital; ${ }^{3}$ Department of Histopathology, Royal Hallamshire Hospital, Sheffield, UK

Introduction Endoscopic ultrasound (EUS) examination and fine needle aspiration (FNA)of the pancreas is now a routine method of obtaining diagnositic tissue for suspected pancreatic mass lesions, however if a biliary self expanding metal stent (SEMS) is in situ it can cast an acoustic shadow and cause local inflammation making EUS visualisation and FNA interpretation more challenging. Despite this it is common for a SEMS to be in place at the time of EUS due to the early treatment required for obstructive jaundice. Conflicting results have been published regarding the success of FNA in the presence of a SEMS, our aim was to analyse the accuracy of pancreatic FNA with or without a SEMS in an hepatobiliary-pancreatic cancer network in the United Kingdom.

Methods A retrospective analysis of all FNAs performed for solid pancreatic lesions in a single centre by 2 endosonographers from August 2010 to December 2012. Patents were identified if they had a biliary SEMS or plastic stent in situ. All FNAs were performed 